\title{
Ultrasonic Diagnosis and Differential Diagnosis of Acute Gastritis
}

\author{
Yan Liu, Xinying Li, Ruizhi Hou* \\ China-Japan Union Hospital of Jilin University, Changchun, Jilin, 130021, China \\ *Corresponding author
}

Keywords: Acute gastritis; Ultrasound diagnosis; Differential diagnosis; Clinical significance.

\begin{abstract}
The paper explored the value of ultrasonic examination in the diagnosis and differential diagnosis of acute gastritis, providing reference for clinical diagnosis and differential diagnosis of acute gastritis. 154 cases of acute gastritis diagnosed by gastroscopy and pathology were selected from January, 2015 to December, 2017 in China-Japan Union Hospital of Jilin University. All the patients were diagnosed by ultrasound. The results of diagnosis and pathology were compared and analyzed. In 154 cases of acute gastritis diagnosed by pathology, 148 cases were diagnosed by ultrasonography, the coincidence rate was 96.1percent, the sensitivity was 84.1 percent, the specificity was 72.7 percent, and the accuracy was 79.22 percent, and the positive predictive value was $80.4 \%$, and the negative predictive value was 77.4 percent. The mucosa of gastric wall in antrum was symmetrical thickened by ultrasound, and the layers were obvious. Echo is strong, and light spot is thicker, as well as gastric peristalsis is normal or a little slow. There was a significant difference from the same appearance of gastric tumor images. In conclusion, Ultrasonographic features of acute gastritis are obvious and can clearly observe the submucosal lesions and peripheral lymph nodes. It is of great value in diagnosis and differential diagnosis.
\end{abstract}

\section{Introduction}

In recent years, with the changes of people's living structure and diet structure, the proportion of gastric lesions has increased obviously, and the diagnosis of gastric lesions has been paid more and more attention in clinic. The traditional examination methods of gastric diseases are mainly gastroscopy, barium radiography and so on. Both traumatic and radiative injuries will affect the patients' compliance, and the prevalence rate is relatively low. Some gastric diseases which are not obvious in clinical symptoms can not be found in time, thus missed the best opportunity of treatment of examination. In addition, gastrography examination has been widely used in recent years. It is easy to operate without special equipment, and has a high degree of acceptance.

\section{Data and Methods}

\subsection{Clinical Data}

From January, 2015 to December, 2017, there are 154 cases of acute gastritis diagnosed by gastroscopy and pathology were selected, including 80 male patients and74 female patients. Their age ranged from 29 to 65 years with an average age of $42.8 \pm 3.5$ years. The patients in both groups were excluded from severe cardiovascular diseases, immune diseases, liver and kidney insufficiency and mental disorders, so as to ensure that the study would not be adversely affected.

\subsection{Methods}

(1) Instrument and equipment. Philips IU22 real-time ultrasonic diagnostic instrument (Philips Electronic Group) was used. The frequency of convex array probe was $2.5-7.6 \mathrm{MHz}$, and $3.5 \mathrm{MHz}$ was generally used. (2) Routine ultrasound examination. Supine position, left supine position, right lateral position, semi-supine position were adopted. Longitudinal standard section, horizontal standard section and oblique standard section were scanned in the area of stomach surface projection. We observed the fundus, body of stomach, antrum, pylorus, duodenum and so on. At the 
same time, we also take the left supine position and high the waist and keep the stomach floor filling, thus facilitating display. Local image amplification or high frequency probe were used to observe the structure of gastric wall, thickness, length, tumor size and surrounding organs, tissue relationship and distant metastasis. The diagnostic criteria of conventional ultrasound: Acute gastritis: The thickness of gastric wall was slightly homogeneous, but the thickness was less than 10 $\mathrm{mm}$, and the layer structure was not clear, and the echo of mucosal layer was low. Mucosal surfaces are rough and uneven or have mucosal bulges. Its height is less than $5 \mathrm{~mm}$. Gastric ulcers: conventional ultrasound observed different depths of indentation in the center of the gastric wall and indentation flat has strong echo spot. Its surrounding gastric wall layer is not very clear, and echo is reduced. Gastric leiomyoma: A solitary bulge of the gastric wall around the lesion was observed by conventional ultrasound with a clear boundary. Gastric cancer: Routine ultrasound observed a localized irregular bulge thickening of the gastric wall. The echo is relatively low, the layer of gastric wall is not very clear, and the gastric mucosa is uneven. The stomach wall is stiff and peristaltic. The boundaries of the surrounding organs are not very clear, and may be accompanied by enlarged lymph nodes around the stomach. (3) Gastrography. The patient pays attention to the light diet 1 day before preparing the gastrography examination, fasting for 8 hours before examination, forbidding drinking for 4 hours, adding the ultrasound aid to the cup with 500 to $600 \mathrm{ml}$ hot water, and then mix well and take warm water. The attention that the child will be reduced, and the examination will begin gradually after 5 to 10 minutes. Patients were routinely examined in the sitting position, supine position and right lateral position so that to conduct examination on cardia, lower esophagus, fundus, body of stomach, antrum, stomach corner and duodenum. According to the characteristics of gastric wall, gastric cavity and perigastric lesions, the reflux condition of contrast agent during peristalsis of gastric wall was examined.

\subsection{Observation indicators}

We should observe the diagnosis of gastric diseases and the TNM staging and pathological results of gastric cancer by gastrography and routine ultrasound. Sensitivity equals to true positive dividing the sum of (true positive plusing false negative); specificity equals to true negative dividing the sum of (true negative plusing false positive); accuracy rate equals to the sum of (true positive plusing true negative) dividing total number of cases.

\subsection{Statistical treatment}

The data were processed by SPSS 19.0 software, and the count data were analyzed by $\chi 2$ test and regarded difference of $\mathrm{P}<0.05$ as statistical significance.

\section{Results}

\subsection{The results of ultrasonic diagnosis in all subjects including in the study}

154 patients were diagnosed as acute gastritis by endoscopy and pathology. The first diagnosis was as follows: 148 cases of acute gastritis, and 4 cases of multiple ulcer and 2 cases of gastric cancer. The accuracy of ultrasonic diagnosis was 79.22 percent with coincidence rate of 96.1 .

\subsection{Ultrasound performance of all subjects in the study}

First, the lesions were mainly located in the antrum, the corner of the stomach and the small curvature of the stomach. The mucosa of the antrum was symmetrically thickened, and the echo was decreased, and the thickness was 5 to $15 \mathrm{~mm}$. Second, the level is obvious, and echo is strong; moreover, light spot is thicker, and gastric cavity is relatively small, and gastric peristalsis is normal or a little slow; third, the mucosal edema was obvious in the lesion location, and the plica was thick, and the surface was not smooth and smooth; there were irregular punctate or flaky hyperechoic features, and the submucosal hyperechoic zone was continuous and intact. Fourth, lymphadenopathy echo appeared around the antrum in some patients. Fifth, after targeted treatment, the above findings were obviously alleviated or disappeared, and there was a significant difference between the above findings and the images of gastric neoplasms. 
According to statistical data, the sensitivity of ultrasound in the diagnosis of acute gastritis was 84.1 percent (74/88), and the specificity was 72.7 percent (48/66). The positive predictive value was 80.4 percent (74/92), and the negative predictive value was 77.4 percent (48/62), and the accuracy was 79.22 percent [(74+48)/154]. (see Table 1$)$.

Table 1 Comparison and analysis of ultrasound and pathological examination

\begin{tabular}{c|c|c|c}
\hline \multirow{2}{*}{ Ultrasound } & \multicolumn{2}{|c|}{ Pathological Examination } & \multirow{2}{*}{ Sum } \\
\cline { 2 - 3 } & + & - & 92 \\
\hline+ & 74 & 18 & 62 \\
\hline- & 14 & 48 & 154 \\
\hline Sum & 88 & 66 & \\
\hline
\end{tabular}

\section{Discussions}

Gastric diseases are common diseases of digestive system. Gastric ulcer and gastric cancer are the most common diseases. Traditional diagnosis mainly depends on X-ray barium meal and gastroscopy. The X-ray barium meal can be used for the whole observation of gastric mucosa, but radiation damage to the human body, and there is a poor taste of the barium agent, and the clinical application of the barium meal is reduced. Gastroscopy biopsy is the main imaging diagnostic method for gastric diseases, and it is also the gold standard for the diagnosis of gastric cancer before operation. However, gastroscopy was invasive and the compliance of the patients was not satisfactory. The method of oral contrast ultrasound (oral contrast ultrasonography, OCUS) was simple, effective, non-invasive and no cross infection. Patient compliance is good. Before examination, the gastric cavity should be filled with oral contrast media, and the gastric cavity should be homogenized by removing the gas and mucus interference, and the gastric wall structure and pathological morphology should be displayed. At present, the value of ultrasonic diagnosis of gastric diseases is more and more recognized and valued by clinicians, and has become one of the hot spots in abdominal ultrasound examination. Ultrasound is one of the most simple methods to observe gastric structure in clinic, but it is difficult to accurately observe the structure and morphology of gastric wall because it is easily disturbed by gas secretion and contents in stomach. Observation of some small lesions is very difficult, interference factors are more. For the diagnosis of gastric diseases, there are gastroscopy and barium radiography, but these methods also have their corresponding shortcomings; gastroscopy not just has certain traumatic, at the same time, hiccup reaction is more serious, so patient has a poor compliance. Although the effect of barium radiography on patients is not very obvious. But barium radiography not only causes radiation damage, but also imposes a physical and psychological burden on patients.

Our country is one of the countries that carried out the application of ultrasonic diagnosis of gastric diseases earlier. Now, gastric ultrasound has been widely used in various gastric diseases, including common and rare diseases such as gastric ulcer, gastric cancer, gastric schwannoma. Diagnosis and differential diagnosis of gastric mucosal prolapse and gastric tuberculosis. Recently, this method has been applied to the screening of gastric diseases in healthy people. Ultrasonic diagnosis of gastric ulcers: Gastric ulcer is one of the most common diseases of the digestive system. Data show that about 10 percent of people suffer from peptic ulcers at some point in their lives.

According to the data of our hospital, 154 cases of acute gastritis were diagnosed by pathology; the coincidence rate of ultrasound diagnosis was 96.1, and the sensitivity was 84.1, and the specificity was 72.7 percent. The accuracy rate was 79.22 , and the positive predictive value was 80.4 percent and the negative predictive value was 77.4 percent. The gastric wall mucosa of antrum showed symmetrical thickening, obvious layer, strong echo with light spot thickening; gastric peristalsis was normal or slightly slow, and there was significant difference with the same appearance of gastric tumor image. Gastric ultrasound can not only observe the changes of gastric mucosa, but also determine the degree of inflammation in submucous tissue, so as to scientifically judge the extent and extent of inflammatory infiltration. Providing effective evidence for clinical 
treatment, ultrasound diagnosis of acute gastritis has the advantages of fast, non-invasive, good reproducibility, especially suitable for the elderly, young children, pregnant women and special groups. To be able to follow up and observe the results of diagnosis and treatment in time, it should be the first choice for the diagnosis of acute gastritis.

To sum up, ultrasonic diagnosis of acute gastritis has obvious features, which can clearly observe the gastric submucosal lesions and surrounding lymph nodes. It has a very high diagnostic value and differential diagnosis value, and can be widely used in clinical.

\section{References}

[1] Fan Xiaohua, Lu Wing. Ultrasonic Diagnosis and Differential Diagnosis of Acute Gastritis [J]. Chinese Journal of Ultrasound Diagnostics, 2005(05): 378-379.

[2] Sangliang, Wang Xuemei. Clinical Significance and Feasibility of Oral Instant Gastrointestinal Ultrasonography in Patients with Stomach Discomfort [J]. Chinese Journal of Medical Ultrasound (Electronic Edition), 2014, 11(12): 977-980.

[3] Meng Fei, Wu Qin. Diagnostic and Differential Diagnosis of Acute Gastritis by Clinical Ultrasound [J]. Modern Digestion and Interventional Diagnosis and Treatment, 2015, 20(04): 376-377.

[4] Wang Junhu, Cao Lili, Chen Xiaojun. Study on the Value of Gastric Contrast-enhanced Ultrasonography in the Diagnosis of Gastric Diseases [J]. Journal of Chinese Medicine, 2015, 12(35): 165-168.

[5] Shen Li, Wang Xiaohong, Wang Yi. Current Situation and Prospect of Ultrasonic Diagnosis of Gastric Diseases in China [J]. Chinese Journal of Medical Ultrasound (Electronic Edition), 2016, 13(06): 401-405.

[6] Chen Jingyi, Xu Liwei. Diagnostic Value of Color Doppler Ultrasonography in Gastroduodenal Tumors [J]. Oncology Clinic of China, 2010, 37(12): 713-715.

[7] Liu Chunli, Wei Kangling. Application Value of Contrast-enhanced Ultrasonography in the Diagnosis of Gastric Disease s [J]. Contemporary Medicine, 2013, 19(14): 57-58. 